\title{
NMR imaging of the soliton lattice profile in the spin-Peierls compound $\mathrm{CuGeO}_{3}$
}

\author{
M. Horvatić ${ }^{1}$, Y. Fagot-Revurat ${ }^{1}$, C. Berthier ${ }^{1,2}$, G. Dhalenne ${ }^{3}$, A. Revcolevschi ${ }^{3}$ \\ ${ }^{1}$ Grenoble High Magnetic Field Laboratory, CNRS and MPI-FKF, B.P. 166, 38042 Grenoble Cedex 09, France \\ ${ }^{2}$ Laboratoire de Spectrométrie Physique, Université J. Fourier, B.P. 87, 38402 Saint-Martin d'Hères, France \\ ${ }^{3}$ Laboratoire de Chimie des Solides, Université de Paris-Sud, 91405, Orsay, France
}

(February 10, 2018)

\begin{abstract}
In the spin-Peierls compound $\mathrm{CuGeO}_{3}$, the commensurate-incommensurate transition concerning the modulation of atomic position and the local spin-polarization is fully monitored at $T=0$ by the application of an external magnetic field $(H)$ above a threshold value $H_{c} \simeq 13$ Tesla. The solitonic profile of the spin-polarization, as well as its absolute magnitude, has been precisely imaged from ${ }^{65} \mathrm{Cu}$ NMR lineshapes obtained for $h=\left(H-H_{c}\right) / H_{c}$ varying from 0.0015 to 2 . This offers a unique possibility to test quantitatively the various numerical and analytical methods developed to solve a generic Hamiltonian in 1-D physics, namely strongly interacting fermions in presence of electron-phonon coupling at arbitrary band filling.

PACS numbers: 75.10.Jm, 75.30.Fv, 75.50.Ee, 76.60.-k
\end{abstract}

The spin-Peierls (SP) transition observed in quantum spin $1 / 2$ antiferromagnetic chains [1] is the magnetic analogue of the well-known Peierls transition in quasi-onedimensional (1D) metallic chains. A 3D array of chains of spins $1 / 2$, in which the position dependent antiferromagnetic exchange interaction $J$ between neighboring spins provides a coupling between spins and lattice distortion, i.e., phonons, undergoes a second order phase transition at $T=T_{S P}$ from a high temperature uniform, paramagnetic and gapless phase, to a low temperature phase in which the lattice is dimerized. The corresponding collective singlet ground state is separated by a gap from triplet excitations and the cost in elastic energy is compensated by the gain in magnetic energy (instead of electronic kinetic energy in the Peierls transition). Applying the canonical Jordan-Wigner transformation, the Hamiltonian of such a chain placed in an external magnetic field $H$ can be mapped onto that of interacting (spinless) fermions coupled to the phonons, $H$ playing the role of the fermionic chemical potential. Physical realizations of SP systems thus offer the unique possibility to check the understanding of this fundamental 1-D Hamiltonian for arbitrary band filling, and particularly close to half-filling (corresponding to $H=0$ ) where commensurability effect are expected to play an important role through the unklapp processes [2]. Until recently, physical realizations of SP system were limited to organic compounds [1]. The recent identification of the first inorganic compound of this type [3], namely $\mathrm{CuGeO}_{3}$, and the synthesis of large and high-purity single crystals, allowing for example inelastic neutron scattering, has therefore raised an important experimental effort [- [n. In particular, one of the key features of the SP systems is the possibility by applying a strong enough magnetic field to cancel the gap between the singlet ground state and the lowest triplet excitation. This induces a transition to a new magnetic phase in which the periodicity of the spin-polarization (i.e., the lo- cal magnetization) and of the associated lattice deformation is incommensurate (IC) with the underlying crystallographic lattice. This commensurate-incommensurate (C-IC) transition takes place by the introduction of a soliton lattice. Each soliton corresponds to a rapid change of phase between two equivalent commensurate (dimerized) domains, and is predicted to carry a total spin $\frac{1}{2}[5]$. Increasing the magnetic field above the threshold value $H_{c}$, the modulation of the lattice distortion and the local spin polarization will continuously evolve from a well-defined soliton lattice to planewave regime.

In this letter, we present a detailed study of ${ }^{65} \mathrm{Cu} \mathrm{NMR}$ lineshapes obtained in a $\mathrm{CuGeO}_{3}$ single crystal in the field range 13-26 $\mathrm{T}$ which gives a direct and detailed access to the real space profile of the spin polarization in the IC phase of $\mathrm{CuGeO}_{3}$ for $h=\left(H-H_{c}\right) / H_{c}$ varying from 0.0015 to 2 . The spin polarization profiles have been successfully parametrized using Jacobi elliptic functions, which are the generic solutions of the sine-Gordon equation, allowing the determination of both the period and the shape of the solitons in the whole range of $h$. These results provide a stringent test for analytical and numerical solutions of the Heisenberg SP Hamiltonian, enabling to investigate the influence of second neighbors interactions, 3-D coupling, or phasons.

In Fig. 1 is shown the evolution of $\mathrm{Cu}$ NMR lineshapes when $H$ is varied from $H_{c}$ to $\simeq 2 H_{c}$. The data are taken on the high frequency satellite of ${ }^{65} \mathrm{Cu}$ isotope in order to avoid overlapping of the lines and ensure maximal resolution. All spectra have been taken at $T=4 \mathrm{~K}$ and for $H$ parallel to the $c$-axis of the single crystal, i.e., along the chains direction. Reducing $T$ to $1.3 \mathrm{~K}$ would increase the width of the spectra by only $5 \%$ while keeping the same lineshape. For values of $H$ close to $H_{c}=13 \mathrm{~T}$, the spectra have been recorded at fixed value of $H$ and sweeping the NMR frequency (Fig. 1, bottom panel), and for higher values of $H$ at fixed frequency sweeping the mag- 
netic field (top panel); the latter method is technically more convenient for wide lines, and is used whenever the modification of the line-shape during the sweep is negligible. Splitting of the high field edge singularity in these spectra is due to slight misalignment of the single crystal. Up to the critical field $H_{c}$, only a narrow symmetric line is seen, corresponding to the homogeneous zero spin polarization in the dimerized phase. Just above, this line is strongly broadened and a wide distribution due to solitons appears. With increasing $H$, the broadened zero spin polarization peak is rapidly suppressed, reflecting the disappearance of the nearly dimerized regions and the approach towards a planewave-like regime.

Knowing the hyperfine and quadrupolar tensors experienced by the copper nuclei in $\mathrm{CuGeO}_{3}$ [6], the resonance frequency of a given copper nucleus at site $i$ on the chain can be directly and precisely converted into the time averaged polarization $\left\langle S_{z}(i)\right\rangle$ of the electronic spin located on that site. To the spatial distribution of $\left\langle S_{z}(i)\right\rangle$ in the IC phase corresponds directly a distribution of local fields $A_{c c}\left\langle S_{z}(i)\right\rangle$, where $A_{c c}$ is the hyperfine coupling constant. This gives rise to a particular NMR lineshape which is nothing but the histogram, or the density distribution of $\left\langle S_{z}(i)\right\rangle$ in absolute units of $\left\langle S_{z}\right\rangle$, thus allowing a determination of the absolute value of the order parameter. For a symmetric and periodic 1-D modulation of the spin polarization, as expected in the IC phase of a SP system, a simple integration of the density distribution directly provides the real space profile over half a period [7]. The NMR lineshape presented in Fig. 11 have however been analyzed somewhat differently, i.e. fitted to the theoretically expected profile of the soliton lattice. In the continuum approximation, the system is described by a phase variable which satisfies a sine-Gordon equation [5,80. The periodic solutions are given in terms of the Jacobi elliptic functions: sn for a lattice distortion and $\mathrm{dn}(\mathrm{cn})$ for the average (staggered) component of the spin polarization. The total spin polarization can then be written as:

$$
\left\langle S_{z}(i)\right\rangle=\frac{W}{2}\left\{\frac{1}{R} \operatorname{dn}[i c /(k \xi), k]+(-1)^{i} \operatorname{cn}[i c /(k \xi), k]\right\}
$$

where $i$ counts the position of the spins in the chain along the $c$ direction, $\xi$ is the correlation length defined by the corresponding sine-Gordon equation, and $R$ is the ratio of amplitudes of the staggered and the average component of spin polarization. $k$ is the modulus of elliptic functions, and $W / 2$ is the amplitude of the staggered component, so that $W$ is just equal to the full span $\left\langle S_{z}\right\rangle_{\max }-\left\langle S_{z}\right\rangle_{\min }$, i.e., to the total width of the NMR spectrum. Here the frequency or magnetic field axis of the spectra is converted to the spin polarization using the known hyperfine coupling constant $\mathrm{A}_{c c}=4.6 \mathrm{~T}$ [6]. When the width of the spectra is known and their integral normalized to unity, the shape of the spectra is determined by only two parameters $R$ and $k$. For each experimental spectrum, these have been determined by the least square fit of the theoretical shape of the spin density distribution (convoluted with a convenient intrinsic NMR linewidth to allow for an experimental resolution) as shown in Fig. 2.

In addition, the period $L$ can be deduced from the NMR line using the fact that it is directly related to the average magnetization $m=g \mu_{B} \overline{\left\langle S_{z}\right\rangle}$ by the relationship $\overline{\left\langle S_{z}\right\rangle} L=1$. Note that this is radically different from usual NMR study of incommensurate phases in dielectrics [9] or CDW systems [10], in which neither the absolute value of the order parameter nor the period can be determined. The summation of $\left\langle S_{z}(x)\right\rangle$ over a period leads to the spatial average spin value $\overline{\left\langle S_{z}\right\rangle}=\pi W / 4 K(k) R$, where $\underline{K(k)}$ is the complete elliptic integral. In Fig. 3 is shown $\overline{\left\langle S_{z}\right\rangle}$ as a function of the magnetic field, together with $1 / L$ directly measured by X-rays, corrected for the anisotropy of the Lande tensor $g$, since $1 / L \propto 1 / g$ [11]. The agreement is very good indeed. However, the range of magnetic field available for X-rays was limited to approximately $4 \%$ above $H_{c}$, and only the vicinity of the C-IC transition could be explored in pure $\mathrm{CuGeO}_{3}$ samples. $\overline{\left\langle S_{z}\right\rangle}$ is found to be proportional to $1 / \ln [8 /(H / H c-1)]$, a function which is raising very sharply at $H_{c}$ and then rapidly becoming proportional to $H$. This is indeed predicted within the mean field theory description [13] and was already found experimentally in the magnetization measurements in other spin-Peierls compounds [i].

In addition to the period $L$, the reconstitution of the spin polarization profile as shown in Fig. 2 allows the determination of the correlation length $\xi=L /[4 k K(k)]$. As a function of magnetic field $\xi$ is somewhat decreasing (Fig. (4). In the field range 1.1-2 $H_{c}$, the values of $\xi / c$ are found equal to 8-7 in agreement with the theoretical predictions. As expected, the amplitude of the spin polarization within the soliton, i.e. the width $W$ is only

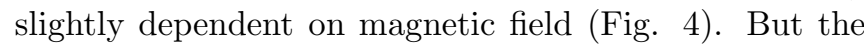
value of the ratio $R$ between the amplitudes of the staggered and the averaged components, which is found to decrease from 1.4 at $H_{c}$ to 1 at about $2 H_{c}$, is definitely several times smaller than that predicted either by analytical [5] or numerical solutions [14,15] of corresponding model Hamiltonians for an isotropic exchange. Although these results are closer to the predictions corresponding to the XY model [12], there is no reason to doubt that the $\mathrm{CuGeO}_{3}$ system is in the Heisenberg limit. Note also that in organic spin-Peierls compounds, the few NMR data available in the incommensurate phase [16, 17] have been interpreted as evidence for a staggered component much larger than the average one, leading to a symmetic lineshape broadening. However, the absence of precise knowledge of the hyperfine coupling, and the poor definition of the lineshapes have precluded more detailed analysis so far.

This strong reduction of the staggered component of spin polarization as observed by NMR and compared to theoretical predictions strongly suggests, if these latter are correct, the existence of some averaging process which 
is not clearly identified yet. Since a static filtering due to a competition between on-site and first-neighbors transferred hyperfine field can be excluded [18], it sounds natural to consider some dynamical process, remembering that NMR only measures time averaged values of the spin polarization. It was recently suggested by Uhrig et al. 14] that this averaging could be due to the zero point motion of phasons, which are acoustic-like excitations always present in incommensurate structures. What is the amplitude of the pinning gap for these excitations, and how they should influence the nuclear relaxations times and the lineshapes in the IC phase remains to be investigated.

A salient result of the analysis is the magnetic field dependence of the shape of the soliton lattice, which is described by the modulus $k$. It turns out that the data for the complementary modulus $k^{\prime}$ follow a power law as a function of reduced field and up to $2 H_{c}$ (our highest experimentally accessible value): $k^{\prime}=\left(1-k^{2}\right)^{1 / 2}=$ $0.56\left(H / H_{c}-1\right)^{0.35}$, which remains to be compared to theoretical predictions. Our experimental $k(H)$ dependence is in clear disagreement to the old prediction based on the Hartree-Fock approximation [13]. This is not surprising, as the customary approximation was to take the Hartree-Fock parameter site-independent, thus reducing the full Heisenberg Hamiltonian to the XY model, which yields to the strong modification of the soliton lattice profile. This error has been realized only recently during the analysis of the $\mathrm{CuGeO}_{3} \mathrm{NMR}$ data [19], and true site-dependent Hartree-Fock approximation approaches indeed the (more) exact solutions, given either by numerics or by the analytical continuum description using bosonization technique.

In conclusion, we have followed the evolution of the soliton lattice as a function of the magnetic field $H$ in the incommensurate magnetic phase of pure $\mathrm{CuGeO}_{3}$ by recording $\mathrm{Cu}$ NMR lineshapes in the field range 13-26 Tesla, i.e. for $h=\left(H-H_{c}\right) / H_{c}$ varying from 0.0015 to 2. Experimental data have been successfully fitted to the analytical solutions of the continuum description, providing for each value of the field both the period $L=1 / \overline{\left\langle S_{z}\right\rangle}$ of the modulation and the width of the solitons $\xi$ [20]. This provides a good overall confirmation of the continuum description of quantum spin $1 / 2$ chains. The amplitude of the staggered magnetization is however found far smaller than predicted by theoretical models, which could be due to the zero point motion of phasons. The magnetic field dependence of $\xi$ should also be a stringent test for numerical or analytical solutions of the spin-Peierls Hamiltonian in presence of frustrating second neighbors coupling and 3D interactions.

We thank S. Buzdin, D. Foerster, D. Poilblanc and G. Uhrig for stimulating discussions. The Laboratoire de Spectrométrie Physique is associé au Centre National de la Recherche Scientifique (UMR 5588) and the GHMFL is Laboratoire conventionné aux Universités J. Fourier et
INPG Grenoble I.

FIG. 1. Magnetic field dependence of ${ }^{65} \mathrm{Cu}$ NMR lineshape in a $\mathrm{CuGeO}_{3}$ single crystal at $4 \mathrm{~K}$ for $h=\left(H-H_{c}\right) / H_{c}$ varying in the range $0.0015-2$. In the bottom panel, the spectra have been recorded by sweeping the frequency. The frequency scales of these spectra are mutually shifted in order to compensate for the variations of magnetic field. In the top panel, the spectra correspond to field sweeps.

FIG. 2. Real space reconstitution of the spin polarization profile (left) and the corresponding fit to Jacobi elliptic functions superposed on experimental spectra (right), as explained in the text. Left top panel shows one period of soliton lattice taken at field only $0.15 \%$ above $H_{c}$, with soliton peaks well separated by "dimerized" regions. High above $H_{c}$ (bottom panels) these regions disappear and solitons strongly overlap making the profile more sinusoidal.

FIG. 3. Field dependence of the period $L$ as deduced directly from ${ }^{65} \mathrm{Cu}$ NMR measurements, compared to the data available from X-rays scattering [11]. For convenience, the same data have been plotted in a linear (left and top) and a logarithmic (right and bottom) scale.

FIG. 4. Magnetic field dependence of the fit parameters for the NMR lineshapes shown in Fig. 1. Top panel shows the full width $W$ (right scale, triangles) of the spectra and the ratio $R$ (left scale, circles) of amplitudes of the staggered (cn) and the average $(\mathrm{dn})$ component of the spin polarization. Bottom panel shows the correlation length $\xi$ (right scale, squares) and the modulus $k^{\prime}$ (left scale, circles) of Jacobi elliptic functions reflecting the evolution of the lattice from the extreme soliton limit to a more sinusoidal profile.

[1] For a review, see W. Bray et al., in Extended Linear Compounds, ed. J. C. Miller (Plenum, New York, 1982), p. 353.

[2] S.A. Brazovski, S.A. Gordyunin and N.N. Kirova, JETP Lett. 31, 456 (1980).

[3] M. Hase, I. Terasaki, and K. Uchinokura, Phys. Rev. Lett. 703651 (1993).

[4] For an overview, see, e.g., J.P. Boucher and L.P. Regnault, J. Phys. I 6, 1939 (1996).

[5] T. Nakano and H. Fukuyama, J. Phys. Soc. Jpn. 49, 1679 (1980); ibidem. 50, 2489 (1981).

[6] Fagot-Revurat et al., Phys. Rev. B 55, 2964 (1997).

[7] Fagot-Revurat et al., Phys. Rev. Lett. 77, 1816 (1996).

[8] J. Zang, S. Chakravarty, A.R. Bishop, Phys. Rev. B 55, R14705 (1997).

[9] R. Blinc, Phys. Rep. 79, 333 (1981). 
[10] C. Berthier et al., in Nuclear Spectroscopy on Charge Density Wave Systems, edited by T. Butz (Kluwer, Dordrecht, 1992), p. 177.

[11] Kiryukhin et al., Phys. Rev. Lett. 76, 4608 (1996).

[12] M. Fujita and K. Machida, J. Phys. C: Solid State Phys. 21, 5813 (1988).

[13] A. I. Buzdin, M. L. Kulic, and V.V. Tugushev, Solid State Commun. 48, 483 (1983).

[14] G.S. Uhrig, F. Schonfeld, J.P. Boucher, Europhys. Lett. 41, 431 (1998); G.S. Uhrig et al., preprint.

[15] Y. Meurdesoif and A. Buzdin, unpublished.

[16] T. W. Hijmans, H. B. Brom, and L. J. de Jongh, Phys. Rev. Lett. 54, 1714 (1985).

[17] S. Brown et al., Phys. Rev. Lett. 80, 5429 (1998).

[18] M. Horvatić et al., Physica B 246-7, 22 (1998).

[19] D. Foerster, Y. Meurdesoif and B. Malet, preprint condmat/9802245.

[20] or alternatively the parameter $k$ of the Jacobi elliptic functions $\mathrm{dn}$ or $\operatorname{cn}(x / k \xi, k)$, where $\xi$ is then equal to $L / 4 k K(k)$. 


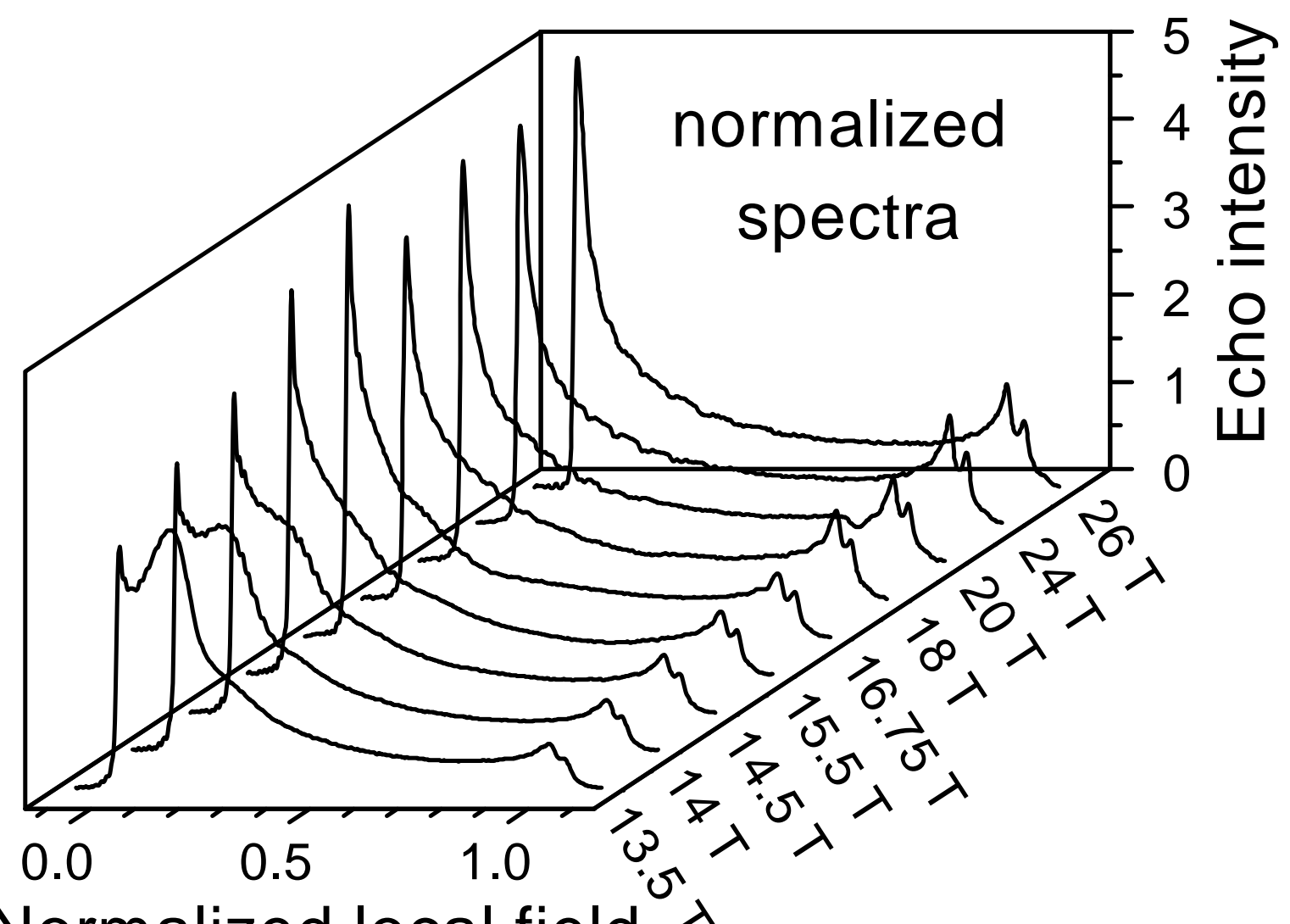

Normalized local field $\lambda$

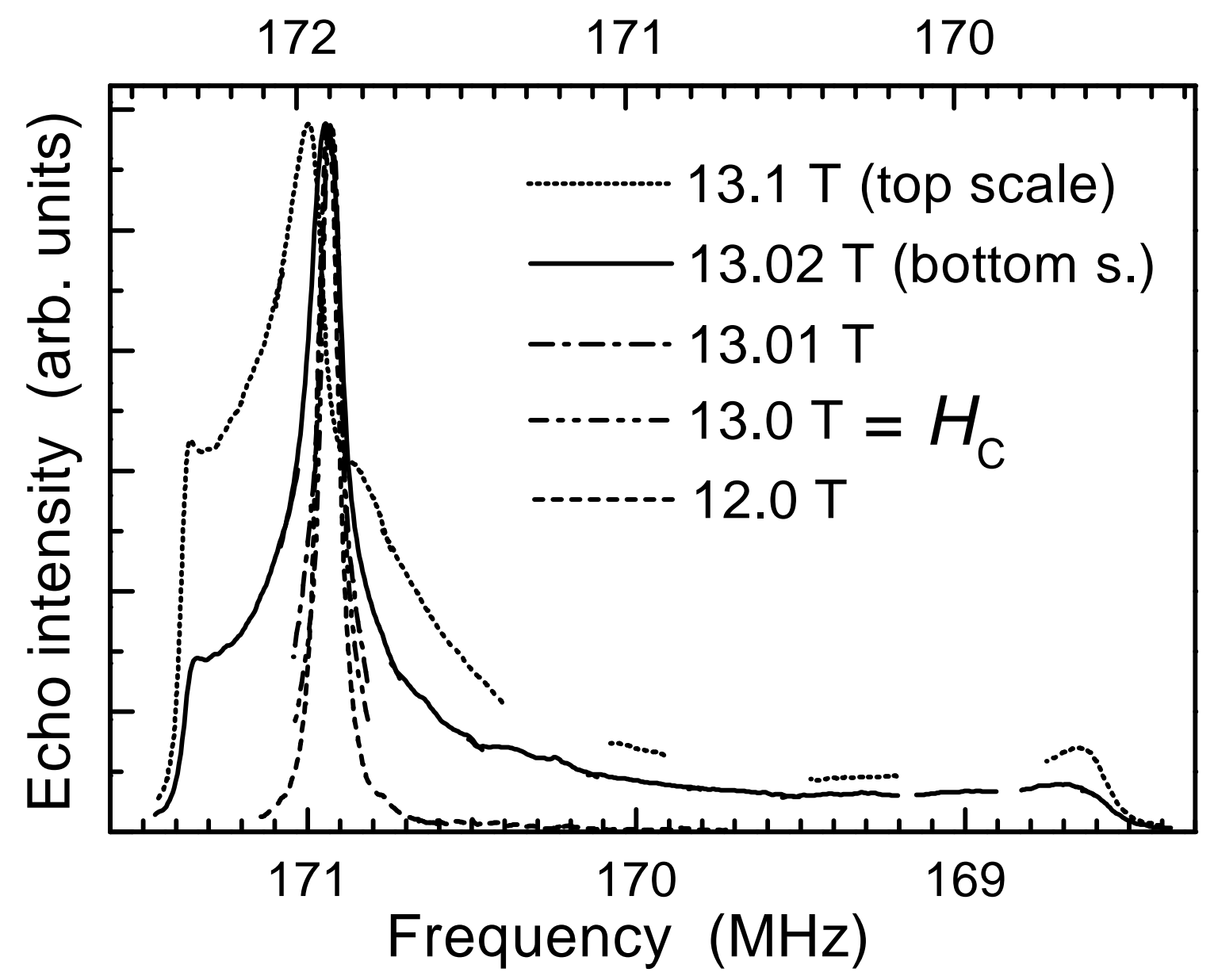

Fig. 1

Phys.Rev.Lett., M. Horvatic et al. 


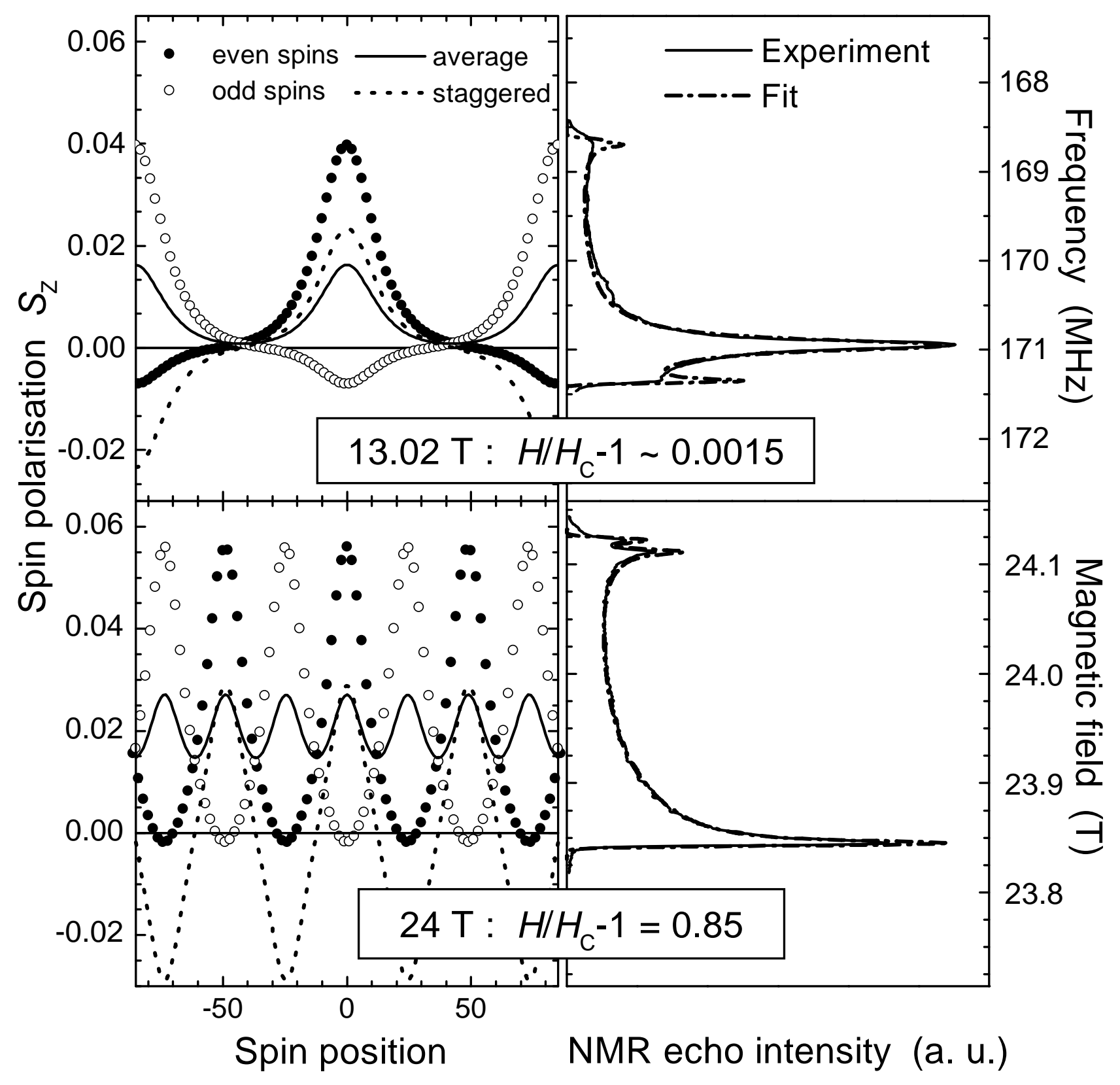

Fig. 2

Phys.Rev.Lett., M. Horvatic et al. 


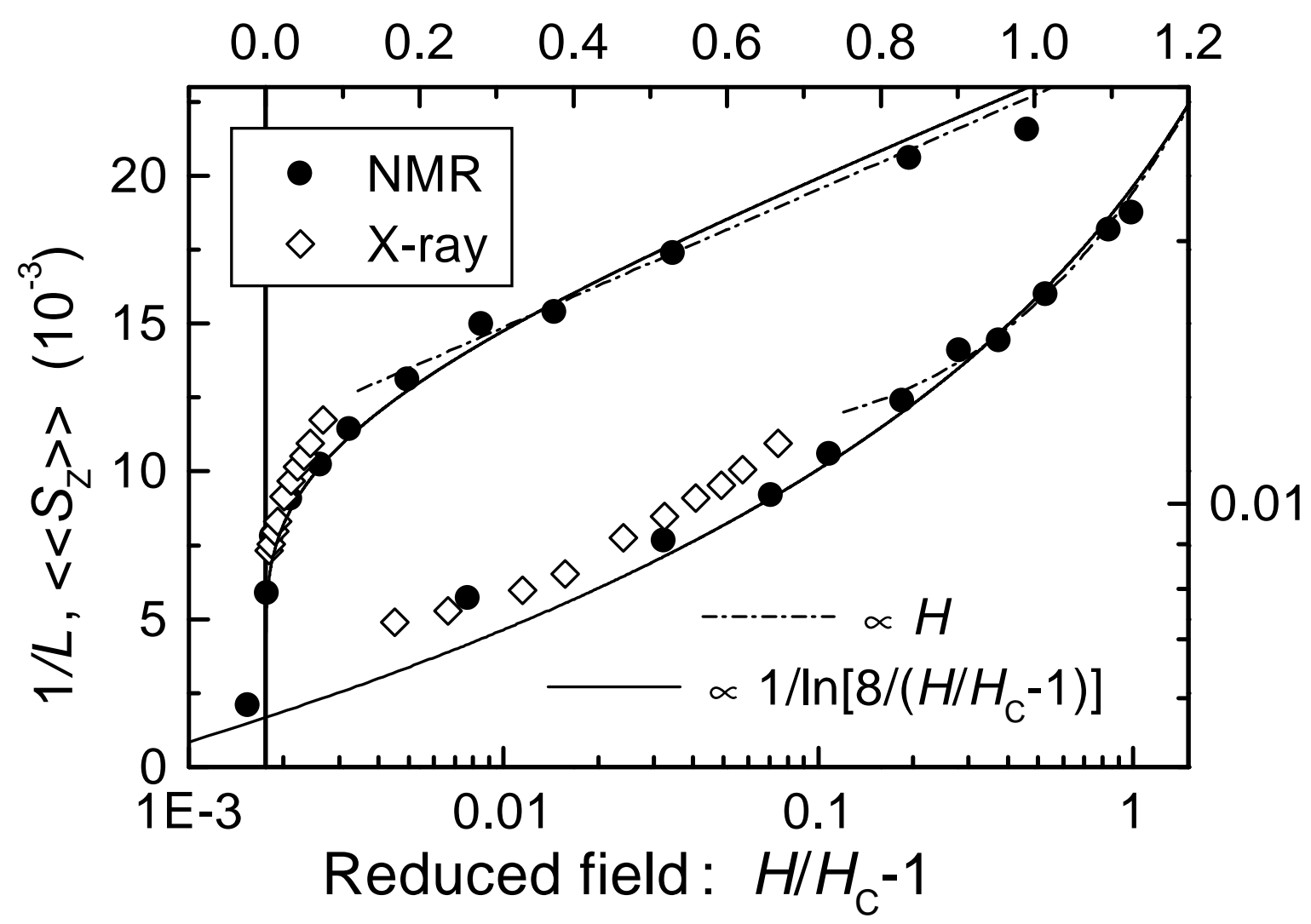

Fig. 3

Phys.Rev.Lett., M. Horvatic et al. 

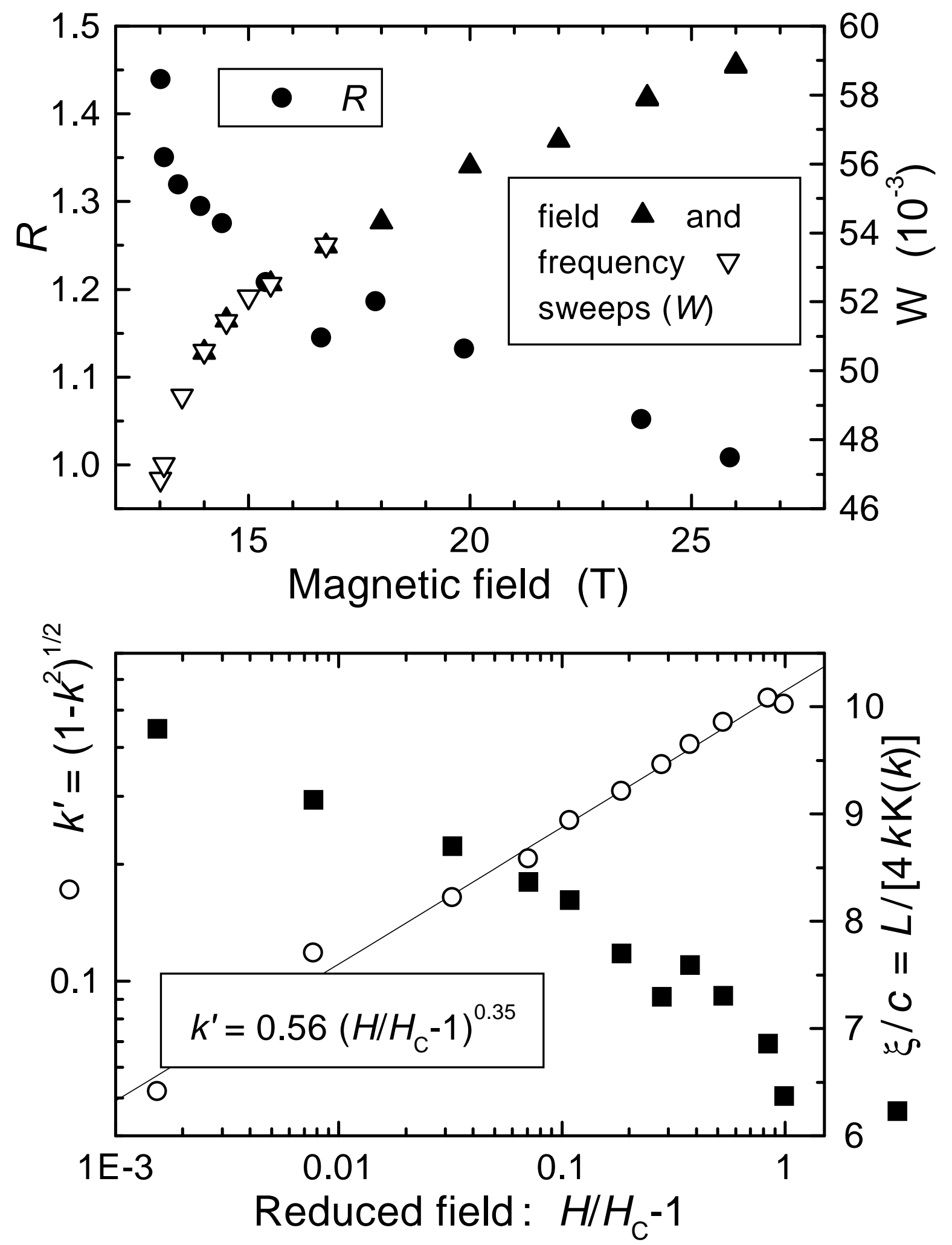

Fig. 4

Phys.Rev.Lett., M. Horvatic et al. 